\title{
The impact of using culture media containing granulocyte-macrophage colony-stimulating factor on live birth rates in patients with a history of embryonic developmental arrest in previous in vitro fertilization cycles
}

\author{
(D) Mehmet Sipahi1', (D) Sezcan Mümüşoğlu², (D) Neslihan Coşkun Akçay², (D) Ayten Sever2, (D) Hande Yeğenoğlu², \\ (D) Gürkan Bozdağ ${ }^{2}$, (D) Lale Karakoç Sökmensüer3 \\ 1Department of Obstetrics and Gynecology, Giresun University Faculty of Medicine, Giresun, Turkey \\ 2Department of Obstetrics and Gynecology, Hacettepe University Faculty of Medicine, Ankara, Turkey \\ 3Department of Histology and Embryology, Hacettepe University Faculty of Medicine, Ankara, Turkey
}

\section{Abstract}

Objective: To investigate the effect of using culture media containing granulocyte-macrophage colony-stimulating factor (GM-CSF) on embryological data and reproductive outcomes in patients with early embryonic developmental arrest.

Material and Methods: Retrospective case-control study. A total of 39 patients, whose embryos were incubated with culture media containing GM-CSF due to embryonic developmental arrest in two previous in vitro fertilization (IVF) cycles in-between January 2016 and November 2017 at Hacettepe University IVF Center, were enrolled. Control group was generated among patients with first IVF attempts due to tubal factor in the same time period. All embryos in the control group were incubated with single step culture medium (without GM-CSF). For the control group selection, matching was done 1:2 ratio considering female age, body mass index, number of M-II oocyte retrieved, and number of embryo transferred $(n=80)$.

Results: Demographic features and embryological data were comparable between two groups. Number of fertilized oocytes (2-pronuclear) was $3.7 \pm 2.0$ in GM-CSF group and $3.9 \pm 2.5$ in the control $(\mathrm{p}=0.576)$. Overall, number of embryos transferred $(1.3 \pm 0.5$ vs $1.3 \pm 0.5$, respectively) and blastocyst transfer rate $(67.6 \%$ vs $59.2 \%$, respectively; $\mathrm{p}=0.401)$ were similar. For the reproductive outcomes, implantation rate $(32.3 \%$ vs $33.1 \%$, respectively; $\mathrm{p}=0.937)$, clinical pregnancy rate $(33.3 \%$ vs $32.5 \%$, respectively; $\mathrm{p}=0.770)$, and live birth rate $(25.2 \%$ vs $26.2 \%$, respectively; $\mathrm{p}=0.943$ ) were similar.

Conclusion: Using GM-CSF-containing culture media in patients with two previous failed IVF attempts due to embryonic developmental arrest might rectify embryological data and reproductive outcomes. To make solid conclusion further randomized controlled trials are warranted. (J Turk Ger Gynecol Assoc 2021; 22: 181-6)

Keywords: GM-CSF, ICSI, recurrent IVF failure, embryonic developmental arrest

Received: 20 September, 2020 Accepted: 16 February, 2021

\section{Introduction}

Up to $20 \%$ of in vitro fertilization (IVF) cycles are cancelled at the stage of follicular growth, oocyte retrieval, fertilization and cleavage stage before embryo transfer (1). Although optimal ovarian stimulation and successful fertilization steps have been completed, some embryos may stop cleaving at the 2to 4-cell stage; this is termed "early embryonic developmental arrest" (2). The rate of early embryonic developmental arrest 
for human embryos in IVF cycles is $\sim 10 \%$, and $40 \%$ of patients experience at least one embryo arrest at the cleavage stage $(2,3)$. The reasons for early embryonic developmental arrest are not fully understood $(4,5)$. Chromosomal abnormalities are observed more often in arrested embryos than normal developed embryos (6). In addition, suboptimal culture conditions, reactive oxygen species (ROS), and inadequate oocyte maturation may also contribute to an increased risk of early embryonic developmental arrest.

Apoptosis is a mechanism for eliminating damaged cells in the human body. However, no biochemical, morphological or structural evidence of apoptosis has been detected in embryos before the 8-cell stage (7). Immature mitochondria in embryos in the 2-to 4-cell stages result in an absence of apoptosis and thus other mechanisms are induced to eliminate defective cells during this period of embryonic development $(8,9)$. It is also known that early cleavage divisions are controlled maternally by transcription factors formed during oogenesis in vivo (10). Therefore, embryos, which are dependent on maternal infrastructure, become more vulnerable to environmental conditions such as ROS in vitro (11). During in vitro culture which negates the protective effect of the oviduct and reduces anti-oxidant defenses (12), lead to poor embryo quality and delayed embryonic development $(5,13)$. Inappropriate expression of the genes encoding Adenosine Deaminase and glucose-6-phosphate dehydrogenase (14) in embryo metabolism and disabled correction of telomere length (15) at these early stages are other options for early embryonic development arrest. Embryo implantation requires embryo-endometrium synchronization, which is a multi-step process regulated by intracellular and intermolecular relations. These interactions are modulated by various cytokines and growth factors via autocrine, paracrine, and endocrine regulations (16). Deficiencies in some interleukins (IL) including IL-1, IL-4, IL-6, colony-stimulating factor-1 (CSF-1), granulocyte-macrophage CSF (GM-CSF), tumor necrosis factor-alpha (TNF- $\alpha$ ), and TNF-b may result in poor embryonic growth, implantation, and pregnancy outcomes $(17,18)$. GM-CSF is a multi-functional cytokine and is synthesized in the epithelial cells of the female reproductive tract, which is essential for modulating stress response genes, heat shock proteins, and apoptosis (19-21). Culture conditions for embryonic development are generally considered suboptimal. The supplementation of these factors may accelerate embryo development, increase blastulation rates, and decrease apoptosis. This study investigated the effects of adding GM-CSF to culture medium on embryological data and reproductive outcomes in patients with previous early embryonic developmental arrest.

\section{Material and Methods}

\section{Patient selection}

All procedures performed in studies involving human participants were in accordance with the ethical standards of the institutional and/or national research committee and with the 1964 Helsinki declaration and its later amendments or comparable ethical standards. Ethical Committee approval was received from Hacettepe University Faculty of Medicine (approval number: 2019/19-02). A signed consent form was obtained from all patients.

For this study, the database of the IVF Centre of Hacettepe University Faculty of Medicine was scrutinized, for the period between January 2016 and November 2017. A total of 49 couples were identified whose embryos were treated with culture media containing GM-CSF (Embryogen ${ }^{\circledR}$, Origio, Denmark) due to previous early embryonic development arrest $(n=49)$. Inclusion criteria were as follows: 1) female $<40$ years old; 2) agonist and antagonist ovarian stimulation cycles; 3) Day 3 or 5 fresh embryo transfer cycles; 4) two consecutive IVF cycles with embryonic development arrest at the 2- to 4-cell cleavage stages. Embryonic development arrest was defined in couples with a history of $\geq 6$ oocytes retrieval and $\geq 5$ fertilized oocytes, but failure to reach embryo transfer during two consecutive IVF cycles. Exclusion criteria included male contributions necessitating surgical sperm retrieval. The inclusion and exclusion criteria narrowed the sample size of study group to 39 patients. To generate a control group from the patients that had undergone IVF treatment in the same time period $(n=80)$ patients with tubal factor, female age $( \pm 1$ year) and antral follicle $( \pm 2)$ were matched in a 1:2 ratio from the database. In the control group, all embryos were incubated in a single step culture medium with human albumin solution (Sage 1-Step, Origio ${ }^{\circledR}$, Denmark).

The primary outcome of this study was investigating the live birth rate (LBR) in patients with a history of recurrent cycle cancelation due to embryonic development arrest after the enrichment of cultural media with GM-CSF. Secondary outcomes were embryo transfer rates on Day 5, implantation rates, and clinical pregnancy rates.

\section{Ovarian stimulation}

Recombinant follicle-stimulating hormone (r-FSH) (Gonal-F; MerckSerono GmbH, Kiel, Germany or Puregon; MSD, Haarlem, the Netherlands) or human menopausal gonadotropin (Merional; IBSA, Lamone, Switzerland, or Menopur, Menogon; Ferring Company, Kiel, Germany) were used solely or in combination for controlled ovarian stimulation. Starting dose varied between 175 and 200 IU. Pituitary suppression was maintained by a GnRH antagonist (Orgalutran; MSD or 
Cetrotide; MerckSerono, addresses as above) or GnRH-agonist protocols, according to physician preference. Cycles were monitored by transvaginal ultrasound.

\section{Laboratory procedure}

Cumulus cell-oocyte complexes (COCs) were accumulated from aspirated follicular fluids under a stereomicroscope. Then COCs were washed twice with Flushing Medium with heparin (Origio) that contained 4-(2-hydroxyethyl)-1-piperazine ethanesulfonic acid. At the end of the oocyte pick up, COCs were cultured in four-well dishes (Thermo Scientific ${ }^{\mathrm{TM}} \mathrm{Nunc}^{\mathrm{TM}}$ ). Each well contained Fert solution (Origio) and was covered with liquid paraffin (Origio). The male patient semen sample was delivered to the laboratory on the day of Ovum Pick-up, and following liquefaction, the sperm sample was evaluated in terms of count, motility, and morphology. Subsequently, sperm sample was prepared using SupraSperm density gradient (Origio) with the "swim-up" technique. Prior to intracytoplasmic sperm injection (ICSI), COCs were removed from cumulus and corona cells using enzymatic (ICSI Cumulase, Origio) and mechanical digestion. After that, the oocytes were assessed in terms of nuclear maturation and all metaphase II (M-II) oocytes underwent ICSI under an inverted microscope with Hoffman optics at x200 magnification. These injected oocytes were cultured under two different conditions. One group $(n=39)$ was cultured with $40 \mu$ l of EmbryoGen (Origio) media until Day 3 , then with BlastGen (Origio) media up to day 5. The others $(n=80)$ were cultured for 3 or 5 days with single step medium containing human albumin solution (Sage 1-Step, Origio). Oocytes were evaluated for fertilization after 16 to 18 hours At 42-44 and 68-72 hours after ICSI, embryos were classified according to Hardarson et al. (22) morphological criteria. Embryo culture was extended to day 5, if there were at least three high quality embryos on day 3 , according to the Gardner criteria (23). After culture with BlastGen or single step media embryos were selected for transfer, were sampled with the Soft-Trans Embryo Transfer Catheter with 15-30 $\mu$ l of media and transferred to the patient under ultrasound.

\section{Statistical analysis}

Mean and standard deviation, or median and $25^{\text {th }}-75^{\text {th }}$ percentiles (interquartile range), were used to describe continuous variables. Pearson chi-square test was used to identify differences between cycle and pregnancy outcomes between the groups. Groups were also compared with independent Samples t-test or Mann-Whitney U test. A twosided p-value $<0.05$ was considered statistically significant. All statistical analyses were performed using SPSS, version 17.0 (IBM Inc., Chicago, IL, USA).

\section{Results}

The demographic characteristics and ovarian reserve tests were similar in the study and control groups (Table 1).

\section{Assisted reproductive technologies cycles in the study and control groups}

GnRH-antagonist protocol was administered to 23 (59\%) of the 39 cycles in women in the study group and 55 (69\%) of the 80 cycles in women in the control group (Table 2). Of the patients undergoing agonist protocols in the study group cycles, four (25\%) were micro-dose flare-up and 12 (75\%) protocols were luteal long leuprolide acetate. In the control group, 5 (20\%) underwent micro-dose flare-up and 20 (80\%) protocols were luteal long leuprolide acetate. On average, the duration of ovulation induction was 9.72 days and 9.70 days for the study and control groups, respectively $(\mathrm{p}=0.961)$. The median $\left(25^{\text {th }}\right.$ $75^{\text {th }}$ percentiles) total $\mathrm{r}$-FSH dose was 2834 IU (1875-3375 IU) in the study group and 2576 IU (1600-3206 IU) in the control group $(p=0.290)$.

\section{Cycle and pregnancy outcomes}

The mean COCs collected were $6.3 \pm 3.0$ and $7.0 \pm 3.0$ in the GM-CSF and control groups, respectively $(p=0.292)$ (Table 2). The respective figure for mean fertilized oocytes (2-pronuclear) was $3.7 \pm 2.0$ and $3.9 \pm 2.5(\mathrm{p}=0.576)$. Mean embryos transferred were $1.3 \pm 0.5$ in both groups. Whereas embryo transfer on Day 5 was available in $67.6 \%$ of patients in the study group, $59.2 \%$ reached the blastocyst stage in the control group $(p=0.401)$. Implantation $(32.3 \%$ vs $33.1 \%, \mathrm{p}=0.937)$, clinical pregnancy rates $(33.3 \%$ vs $32.5 \%, \mathrm{p}=0.770)$, and LBRs $(25.2 \%$ vs $26.2 \%$, $\mathrm{p}=0.943$ ) were comparable between groups.

\section{Discussion}

According to the İstanbul consensus, embryos reach 7-9 cells by Day 3, with fragmentation under 15\% and no multi-nucleation,

Table 1. Demographic characteristics of both groups

\begin{tabular}{|l|l|l|l|}
\hline & $\begin{array}{l}\text { GM-CSF } \\
\text { group } \\
(\mathbf{n = 3 9 )}\end{array}$ & $\begin{array}{l}\text { Control } \\
\text { group } \\
(\mathbf{n = 8 0 )}\end{array}$ & $\mathbf{p}$ \\
\hline Female age, (years) & $32.8 \pm 4.1$ & $31.7 \pm 5.3$ & 0.219 \\
\hline $\mathrm{AFC},(\mathrm{n})$ & $12.2 \pm 8.3$ & $12.9 \pm 8.0$ & 0.412 \\
\hline $\mathrm{AMH},(\mathrm{ng} / \mathrm{mL})$ & $2.38 \pm 1.6$ & $2.30 \pm 1.6$ & 0.800 \\
\hline BMI, (kg/m $\left.{ }^{2}\right)$ & $25.1 \pm 4.2$ & $24.8 \pm 4.5$ & 0.739 \\
\hline $\begin{array}{l}\text { Duration of infertility, } \\
\text { (months) }\end{array}$ & $75.1 \pm 55.5$ & $57.7 \pm 39.6$ & 0.085 \\
\hline $\begin{array}{l}\text { Data are expressed as means } \pm \text { SD, p<0.05. } \\
\text { GM-CSF: Granulocyte-macrophage colony-stimulating factor, AFC: Antral } \\
\text { follicle count, AMH: Anti-Müllerian hormone, BMI: Body mass index, SD: } \\
\text { Standard deviation }\end{array}$ & \multicolumn{4}{l}{} \\
\hline
\end{tabular}


Table 2. Comparison of cycle characteristics and pregnancy outcomes of both groups

\begin{tabular}{|l|l|l|l|}
\hline & $\begin{array}{l}\text { GM-CSF } \\
\text { group (n=39) }\end{array}$ & $\begin{array}{l}\text { Control group } \\
\text { (n=80) }\end{array}$ & $\mathbf{p}$ \\
\hline $\begin{array}{l}\text { Antagonist protocol } \\
\text { (n, \%) }\end{array}$ & $23 / 39(59)$ & $55 / 80(69)$ & 0.309 \\
\hline Total r-FSH dose (IU) & $2834(1875-3375)$ & $2576(1600-3206)$ & 0.290 \\
\hline $\begin{array}{l}\text { Cycle cancelation } \\
\text { rate (n, \%) }\end{array}$ & $5 / 39(12.8)$ & $9 / 80(11.2)$ & 0.802 \\
\hline $\begin{array}{l}\text { Number of retrieved } \\
\text { oocytes }\end{array}$ & $6.3 \pm 3.0$ & $7.0 \pm 3.0$ & 0.292 \\
\hline $\begin{array}{l}\text { Number of M-II } \\
\text { oocytes }\end{array}$ & $4.9 \pm 2.6$ & $5.6 \pm 3.5$ & 0.263 \\
\hline Number of 2PN & $3.7 \pm 2.0$ & $3.9 \pm 2.5$ & 0.576 \\
\hline $\begin{array}{l}\text { Number of embryos } \\
\text { transferred }\end{array}$ & $1.3 \pm 0.5$ & $1.3 \pm 0.5$ & 0.707 \\
\hline $\begin{array}{l}\text { Embryo transfer day } \\
\text { (n, \%) }\end{array}$ & - & - & 0.416 \\
\hline Day 3 & $11 / 34(32.4)$ & $29 / 71$ (40.8) & - \\
\hline Day 5 & $23 / 34(67.6)$ & $42 / 71(59.2)$ & - \\
\hline $\begin{array}{l}\text { Implantation rate, } \\
\text { (\%) }\end{array}$ & $32.3 \pm 44.2$ & $33.1 \pm 46.2$ & 0.937 \\
\hline $\begin{array}{l}\text { Clinical pregnancy } \\
\text { rate, (n, \%) }\end{array}$ & $13 / 39(33.3)$ & $26 / 80(32.5)$ & 0.770 \\
\hline $\begin{array}{l}\text { Miscarriage rate, } \\
\text { (n, \%) }\end{array}$ & $3 / 13(23.1)$ & $5 / 26(19.2)$ & 0.9973 \\
\hline Live birth rate, (\%) & $10 / 39(25.6)$ & $21 / 80(26.2)$ & SD: \\
\hline $\begin{array}{l}\text { Data are expressed as } \\
\text { percentages. } \\
\text { GM-CSF: Granulocyte-macrophage colony-stimulating factor, } \\
\text { Recombinant follicle-stimulating hormone, } \\
\text { Standard deviation }\end{array}$ & & & M-II: Metaphase \\
\hline
\end{tabular}

and have cleaved during the preceding 24 hours are called "normal", embryos that have 6 or fewer cells on Day $3(68 \pm 1$ hour post insemination), but have cleaved during the former 24 hours are called "Slow", and embryos that have not cleaved within 24 hours are called "Arrested" (24). Approximately $40 \%$ of couples that undergo treatment exhibit at least one embryonic arrest per treatment cycle and $10-15 \%$ of all human embryos arrest at the early cleavage stage $(2,3)$. Despite the sufficient number of oocytes and zygotes, embryonic development arrest is closely associated with assisted reproductive technology (ART) and may lead to recurrent treatment failure. The presence of arrested embryos may diminish the total number of available embryos and affect the treatment cycle outcome. This condition is destructive for the patients receiving treatment. The reasons for early embryonic developmental arrest are not fully understood (4,5). Chromosomal abnormalities, suboptimal culture conditions, ROS, apoptosis and inadequate oocyte maturation have all been shown to be a reason for early embryonic developmental arrest (6).
Suboptimal culture conditions are a possible cause of early embryonic development arrest $(4,5)$. Culture medium and ingredients are crucial for embryo development and implantation. Therefore, various growth factors and cytokines have been examined by supplementing the culture medium to optimize embryo development. GM-CSF may be an ideal candidate for embryo development owing to its natural existence in the surface of the fallopian tubes and endometrial cells (19-21). GM-CSF initiates biological activity by binding to its receptor, which is a $18-22 \mathrm{kDa}$ glycoprotein and is a cytokine/growth factor secreted by the uterine epithelium and oviducts under the influence of estrogens. GM-CSF acts as a principal mediator of recruitment and activation of neutrophils and macrophages during early pregnancy (19). GM-CSF also activates granulocytes, mononuclear phagocytes and dendritic cells, modulates their migration into tissues and activity in situ, and supports cytotoxicity, phagocytosis, antigen introduction, and cytokine discharge (25). GM-CSF is involved in embryonic development, growth and viability by effecting cell proliferation, blastocyst development, hatching and implantation (26). Some genetic combinations may result in the production of polymorphic Killer immunoglobulin-like receptors (KIR) genes from maternal decidual Natural Killer cells and defective human leukocyte antigen-C (HLA-C) genes from fetal trophoblasts. Incompatibility between KIR-HLA-C can cause abnormal placentation. GM-CSF also provides higher KIR-HLA-C coupling by increasing the migration of primary trophoblasts (27).

Culture media containing GM-CSF accelerates embryo development, increases early cleavage embryo counts that develop at the blastocyst stage, and increases viable inner mass cells with less apoptosis in cultured human embryos in vitro (28). Tevkin et al. (29) reported that using GM-CSF in the culture medium, to improve recurrent ART failure, increased, although not statistically significantly, the clinical pregnancy rates compared to the control group ( $39.1 \%$ vs $27.8 \%)$. The implantation rate after seven weeks of gestation $(11.6 \%$ vs $20.4 \%, \mathrm{p}<0.05)$ and clinical pregnancy rate after first trimester $(9.1 \%$ vs $17.4 \%, p<0.001)$ were significantly higher in the GMCSF group (29). They concluded that adding GM-CSF to the cultural medium improved implantation and enhanced the success of IVF/ICSI cycles (29).

Culture medium with GM-CSF was used in a randomized clinical trial to compare the effect of low $(2 \mathrm{mg} / \mathrm{mL})$ and high (5 $\mathrm{mg} / \mathrm{mL}$ ) human serum albumin (HSA) (28). Under the low HSA concentration, the ongoing implantation rate at first trimester for the GM-CSF group was significantly higher (23.5\% vs $16.7 \%, \mathrm{p}=0.007$ ) than the control group, whereas the high HSA concentrations were similar between groups $(22.4 \%$ vs 
$21.1 \%, p=0.53)$, respectively. Overall, LBRs were significantly higher in the GM-CSF group compared to the control group ( $28.9 \%$ vs $24.1 \%, p=0.03$ ). Also, failed embryonic development in previous cycles in culture medium with high HSA, implantation, and clinical pregnancy rates were similar in the subsequent cycle with GM-CSF-supplemented culture medium compared to the control group (28). Also, in this study in terms of reproductive outcomes, implantation rates $(32.3 \%$ vs $33.1 \%$ respectively; $\mathrm{p}=0.937$ ), clinical pregnancy rate $(33.3 \%$ vs $32.5 \%$, respectively; $\mathrm{p}=0.770)$ and LBR ( $25.2 \%$ vs $26.2 \%$, respectively; $\mathrm{p}=0.943$ ) were the same.

The effect of culture media enriched with GM-CSF, heparinbinding epidermal growth factor-like factor, and leukemia inhibitory factor has been investigated in ICSI cycles and the ongoing pregnancy rates (30). Ongoing pregnancy rates were higher in the group using enriched culture media [106/224 (47\%) vs 78/219 (36\%); absolute rate difference (ARD): 12 ; 95\% confidence interval (CI), 2.5-21]. Cumulative LBRs were also higher [132/224 (60\%) vs 97/219 (44\%); ARD: 12; 95\% CI, 4-20], and pregnancy loss rates were lower [27/124 (22\%) vs $37 / 103$ (36\%); ARD: $-14 ; 95 \% \mathrm{CI},-26$ to -2 ]. The authors argued that less biochemical stress occured in the enriched culture environment, so that less energy was consumed by embryo plasticity, and the reserve was then available for postimplantation development.

In a retrospective study using GM-CSF in culture medium in fresh transfer cycles, the cleavage rate and the blastocyst formation rate were found to be similar compared to the control group (31). However, the available embryo rate was significantly higher in the GM-CSF group. In the subgroup analysis, cleavage rate and blastocyst formation rate were found to be significantly higher in those over 38 years old.

In a review article evaluating the effects of GM-CSF in subfertile patients undergoing ART, the beneficial effects of cytokine supplementation have been observed in periods of embryonic development. However, the implantation rates and pregnancy rates were different among investigated studies. According to a Cochrane meta-analysis, there was no evidence that GM-CSF supplemented culture media had any superiority compared to standard media in terms of clinical outcomes (32). Furthermore, only one large, randomized, controlled trial ended with positive results in LBRs between the study and control groups (26). Similarly, in our study the number of M-II and fertilized oocytes were comparable between the groups. Retrospective design and a small sample size were limitations of this study.

GM-CSF supplemented culture medium might be recommended to patients with a history of recurrent failed IVF attempts due to arrest in embryo development.

\section{Conclusion}

Using GM-CSF containing culture media in patients with two previous failed IVF attempts due to embryonic developmental arrest might rectify embryological data and reproductive outcomes. However, further studies are needed to confirm these findings and support the routine use of GM-CSF supplemented culture medium in IVF.

Ethics Committee Approval: All procedures performed in studies involving human participants were in accordance with the ethical standards of the institutional and/or national research committee and with the 1964 Helsinki declaration and its later amendments or comparable ethical standards. Ethical Committee approval was received from Hacettepe University Faculty of Medicine (approval number: 2019/19-02).

Informed Consent: A signed consent form was obtained from all patients.

Peer-review: Externally peer-reviewed.

Author Contributions: Surgical and Medical Practices: G.B., S.M., N.C.A., H.Y., A.S.; Concept - L.K.S., N.C.A., H.Y., A.S.; Design - G.B., S.M., M.S.; Data Collection or Processing - M.S.; Analysis or Interpretation - M.S., S.M.; Literature Search - M.S., N.C.A.; Writing - M.S., N.C.A., S.M.

Conflict of Interest: No conflict of interest is declared by the authors.

Financial Disclosure: The authors declared that this study received no financial support.

\section{References}

1. Johnston-MacAnanny EB, DiLuigi AJ, Engmann LL, Maier DB, Benadiva CA, Nulsen JC. Selection of first in vitro fertilization cycle stimulation protocol for good prognosis patients: gonadotropin releasing hormone antagonist versus agonist protocols. J Reprod Med 2011; 56: 12-6.

2. Memili E, First NL. Zygotic and embryonic gene expression in cow: a review of timing and mechanisms of early gene expression as compared with other species. Zygote 2000; 8: 87-96.

3. Betts DH, Madan P. Permanent embryo arrest: molecular and cellular concepts. Mol Hum Reprod 2008; 14: 445-53.

4. Hardy K, Spanos S, Becker D, Iannelli P, Winston RM, Stark J. From cell death to embryo arrest: mathematical models of human preimplantation embryo development. Proc Natl Acad Sci U S A 2001; 98: 1655-60

5. Hu DB, Li ZS, Ali I, Xu LJ, Fang NZ. Effect of potential role of p53 on embryo development arrest induced by $\mathrm{H} 2 \mathrm{O} 2$ in mouse. In Vitro Cell Dev Biol Anim 2017; 53: 344-53.

6. Almeida PA, Bolton VN. Cytogenetic analysis of human preimplantation embryos following developmental arrest in vitro. Reprod Fertil Dev 1998; 10: 505-13. 
7. Hardy K. Apoptosis in the human embryo. Rev Reprod 1999; 4: 12534.

8. Van Blerkom J. Mitochondria in human oogenesis and preimplantation embryogenesis: engines of metabolism, ionic regulation and developmental competence. Reproduction 2004; 128: 269-80

9. Brad AM, Hendricks KE, Hansen PJ. The block to apoptosis in bovine two-cell embryos involves inhibition of caspase-9 activation and caspase-mediated DNA damage. Reproduction 2007; 134: 78997.

10. Braude P, Bolton V, Moore S. Human gene expression first occurs between the four- and eight-cell stages of preimplantation development. Nature 1988; 332: 459-61.

11. Betts DH, King WA. Genetic regulation of embryo death and senescence. Theriogenology 2001; 55: 171-91.

12. Guérin P, El Mouatassim S, Ménézo Y. Oxidative stress and protection against reactive oxygen species in the pre-implantation embryo and its surroundings. Hum Reprod Update 2001; 7: 175-89.

13. McPherson NO, Zander-Fox D, Lane M. Stimulation of mitochondrial embryo metabolism by dichloroacetic acid in an aged mouse model improves embryo development and viability. Fertil Steril 2014; 101: 1458-66.

14. Taylor DM, Ray PF, Ao A, Winston RM, Handyside AH. Paternal transcripts for glucose-6-phosphate dehydrogenase and adenosine deaminase are first detectable in the human preimplantation embryo at the three- to four-cell stage. Mol Reprod Dev 1997; 48: 442-8.

15. Liu L, Bailey SM, Okuka M, Muñoz P, Li C, Zhou L, et al. Telomere lengthening early in development. Nat Cell Biol 2007; 9: 1436-41.

16. Segerer S, Kammerer U, Kapp M, Dietl J, Rieger L. Upregulation of chemokine and cytokine production during pregnancy. Gynecol Obstet Invest 2009; 67: 145-50.

17. Laird SM, Tuckerman EM, Cork BA, Linjawi S, Blakemore AI, Li TC. A review of immune cells and molecules in women with recurrent miscarriage. Hum Reprod Update 2003; 9: 163-74.

18. Scarpellini F, Sbracia M. Use of granulocyte colony-stimulating factor for the treatment of unexplained recurrent miscarriage: a randomised controlled trial. Hum Reprod 2009; 24: 2703-8.

19. Robertson SA, Mayrhofer G, Seamark RF. Uterine epithelial cells synthesize granulocyte-macrophage colony-stimulating factor and interleukin-6 in pregnant and nonpregnant mice. Biol Reprod 1992; 46: 1069-79.

20. Chin PY, Macpherson AM, Thompson JG, Lane M, Robertson SA. Stress response genes are suppressed in mouse preimplantation embryos by granulocyte-macrophage colony-stimulating factor (GM-CSF). Hum Reprod 2009; 24: 2997-3009.

21. Giacomini G, Tabibzadeh SS, Satyaswaroop PG, Bonsi L, Vitale L, Bagnara GP, et al. Epithelial cells are the major source of biologically active granulocyte macrophage colony-stimulating factor in human endometrium. Hum Reprod 1995; 10: 3259-63.

22. Hardarson T, Hanson C, Sjögren A, Lundin K. Human embryos with unevenly sized blastomeres have lower pregnancy and implantation rates: indications for aneuploidy and multinucleation. Hum Reprod 2001; 16: 313-8.

23. Gardner DK, Schoolcraft WB. In-vitro culture of human blastocyst. In: Jansen R, Mortimer D, editors. Towards reproductive certainty: infertility and genetics beyond Parthenon Press, Carnforth; 1999. p. 377-88.

24. Alpha Scientists in Reproductive Medicine and ESHRE Special Interest Group of Embryology. The Istanbul consensus workshop on embryo assessment: proceedings of an expert meeting. Hum Reprod 2011; 26: 1270-83.

25. Crosier PS, Lewis PM, Hall LR, Vitas MR, Morris CM, Beier DR, et al. Isolation of a receptor tyrosine kinase (DTK) from embryonic stem cells: structure, genetic mapping and analysis of expression. Growth Factors 1994; 11: 125-36.

26. Siristatidis C, Vogiatzi P, Salamalekis G, Creatsa M, Vrachnis N, Glujovsky D, et al. Granulocyte macrophage colony stimulating factor supplementation in culture media for subfertile women undergoing assisted reproduction technologies: a systematic review. Int J Endocrinol 2013; 2013: 704967.

27. Cruz M, Alecsandru D, García-Velasco JA, Requena A. Use of granulocyte colony-stimulating factor in ART treatment does not increase the risk of adverse perinatal outcomes. Reprod Biomed Online 2019; 39: 976-80.

28. Ziebe S, Loft A, Povlsen BB, Erb K, Agerholm I, Aasted M, et al. A randomized clinical trial to evaluate the effect of granulocytemacrophage colony-stimulating factor (GM-CSF) in embryo culture medium for in vitro fertilization. Fertil Steril 2013; 99: 1600-9.

29. Tevkin S, Lokshin V, Shishimorova M, Polumiskov V. The frequency of clinical pregnancy and implantation rate after cultivation of embryos in a medium with granulocyte macrophage colonystimulating factor (GM-CSF) in patients with preceding failed attempts of ART. Gynecol Endocrinol 2014; (Suppl1): 9-12.

30. Fawzy M, Emad M, Elsuity MA, Mahran A, Abdelrahman MY, Fetih AN, et al. Cytokines hold promise for human embryo culture in vitro: results of a randomized clinical trial. Fertil Steril 2019; 112: 849-57.e1.

31. Chu D, Fu L, Zhou W, Li Y. Relationship between granulocytemacrophage colony-stimulating factor, embryo quality, and pregnancy outcomes in women of different ages in fresh transfer cycles: a retrospective study. J Obstet Gynaecol 2020; 40: 626-32.

32. Armstrong S, MacKenzie J, Woodward B, Pacey A, Farquhar C. GM-CSF (granulocyte macrophage colony-stimulating factor) supplementation in culture media for women undergoing assisted reproduction. Cochrane Database Syst Rev 2020; 7: CD013497. 